\title{
Rapid identification of allergenic and pathogenic molds in environmental air by an oligonucleotide array
}

\author{
Wen-Tsung Hung ${ }^{1}$, Shu-Li Su², Lin-Yi Shiu ${ }^{1}$ and Tsung C Chang ${ }^{2 *}$
}

\begin{abstract}
Background: Airborne fungi play an important role in causing allergy and infections in susceptible people. Identification of these fungi, based on morphological characteristics, is time-consuming, expertise-demanding, and could be inaccurate.
\end{abstract}

Methods: We developed an oligonucleotide array that could accurately identify 21 important airborne fungi (13 genera) that may cause adverse health problems. The method consisted of PCR amplification of the internal transcribed spacer (ITS) regions, hybridization of the PCR products to a panel of oligonucleotide probes immobilized on a nylon membrane, and detection of the hybridization signals with alkaline phosphatase-conjugated antibodies.

Results: A collection of 72 target and 66 nontarget reference strains were analyzed by the array. Both the sensitivity and specificity of the array were 100\%, and the detection limit was $10 \mathrm{pg}$ of genomic DNA per assay. Furthermore, 70 fungal isolates recovered from air samples were identified by the array and the identification results were confirmed by sequencing of the ITS and D1/D2 domain of the large-subunit RNA gene. The sensitivity and specificity of the array for identification of the air isolates was 100\% (26/26) and 97.7\% (43/44), respectively.

Conclusions: Identification of airborne fungi by the array was cheap and accurate. The current array may contribute to decipher the relationship between airborne fungi and adverse health effect.

\section{Background}

Fungi are widely distributed in the natural environment. Fungal spores can be easily dispersed into the air and may cause serious health problems. Exposure to fungal spores can cause a wide spectrum of allergenic reactions, such as asthma, and infections in susceptible individuals [1-4]. Asthma prevalence has considerably increased in recent decades such that it is now one of the most common chronic disorders in the world [5-7]. Some severe diseases, such as allergic bronchopulmonary aspergillosis and fungal sinusitis, may be found in susceptible or immunocompromised individuals through mold exposure $[8,9]$. The predominant genera of airborne fungi causing health concern are Alternaria, Aspergillus, Cladosporium, and Penicillium [4].

\footnotetext{
* Correspondence: tsungcha@mail.ncku.edu.tw

${ }^{2}$ Department of Medical Laboratory Science and Biotechnology, College of Medicine, National Cheng Kung University, Tainan, Taiwan Full list of author information is available at the end of the article
}

In order to decipher the relationship between fungi and potential fungal infection, it is imperative to establish methods that can accurately identify airborne fungi to the species level and the method could be easily followed. Conventional methods for fungal identification are primarily based on morphological and physiological tests [10]. These tests often require several days or even weeks and the results can be inconclusive or inaccurate [11]. Even for a mycologist, the identification of airborne fungi to the species level can be challenging, due to the taxonomically high divergence of these microorganisms. In recent years, numerous DNA-based methods have been developed to identify a variety of medically important fungi [12]. The rRNA genes have been extensively used as the targets for molecular identification $[12,13]$. These methods include DNA probes [14], PCR-restriction enzyme analysis [15], real-time PCR [16], and DNA sequencing $[17,18]$. PCR techniques are particularly promising because of their simplicity, sensitivity, and
C Biomed Central 
specificity. However, these methods can identify only one or a limited number of species at a time.

A variety of DNA array methods, having the capacity to simultaneously identify multiple targets, have been developed to identify pathogenic fungi [19-23,26,30] with high sensitivity and specificity. In contrast, literatures using the array platform to detect airborne fungi are very limited and so far only one study using nonspecific probes was reported [24]. In our previous studies, oligonucleotide probes designed from the internal transcribed spacer (ITS) regions have been developed to identify a wide variety of pathogenic molds $(19,26)$ and yeasts $(30)$, including some airborne species. The aim of this study was to expand the probe panel to identify 21 airborne fungal species (13 genera) that may cause health problems in susceptible persons.

\section{Methods}

Fungal strains

A total of 73 target strains (strains we aimed to identify) representing 21 species (13 genera) (Table 1) and 66 nontarget strains (66 species, additional file 1) were used in this study. These strains were obtained from the Bioresources Collection and Research Center (BCRC, Hsinchu, Taiwan), the American Type Culture Collection (ATCC,
Manassas, Virginia, USA), and Centraalbureau voor Schimmelcultures (CBS, Utrecht, The Netherlands).

\section{DNA extraction}

Mycelia (approximately $0.5 \times 0.5 \mathrm{~cm}$ ) grown on Saubouraud dextrose agar were transferred into a 2-ml screw cap tube (Azygen Sientific, Union City, California, USA) containing $300 \mathrm{mg}$ of zirconium/silica beads $(0.5 \mathrm{~mm}$ in diameter, Biospec Products, Bartlesville, Oklahoma, USA) in $1 \mathrm{ml}$ of sterilized saline. The mycelial suspension was shaken in a cell disrupter (Mini-Beadbeater, Biospec Products) for $5 \mathrm{~min}$ at a speed of 4,200 rpm. An $0.1 \mathrm{ml}$ aliquot of the disrupted cell suspension was transferred to a 1.5- $\mathrm{ml}$ centrifuge tube and centrifuged at $8,000 \times g$ for 10 min. Fungal DNA in the supernatant was extracted by a DNA extraction kit (Viogene, Taipei, Taiwan) following the manufacturer's instructions [19].

\section{ITS amplification and sequencing}

The fungus-specific universal primers ITS1 (5'-TCCGTAG GTGAACCTGCGG-3') and ITS5 (5'-GGAAGTAAAAGT CGTAACAAGG-3') were used as the forward primers [25], while ITS4 (5'-TCCTCCGCTTAT TGATATGC-3') was used as the reverse primer to amplify the ITS region [25]. PCR was performed in a total reaction volume of

Table 1 Fungal strains used for identification by the oligonucleotide array

\begin{tabular}{|c|c|c|}
\hline Species & Strain $^{a}$ & Total no. of strains \\
\hline Acremoniun strictum & BCRC 32290, BCRC $32239^{\top}$ & 2 \\
\hline Alternaria alternata & BCRC 32888, BCRC 30501, CBS 105.49 & 3 \\
\hline Aspergillus flavus & BCRC 30006, BCRC 30007, BCRC 30008, BCRC 30009, BCRC 30165' & 5 \\
\hline Aspergillus fumigatus & BCRC 32149, BCRC 30502', BCRC 33373, BCRC 33380, BCRC 33381 & 5 \\
\hline Aspergillus niger & BCRC 320201, BCRC 30204, BCRC 31130', BCRC 30507, BCRC 32720, BCRC 33046 & 6 \\
\hline Aspergillus versicolor & 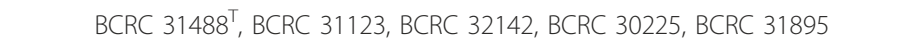 & 5 \\
\hline Aureobasidium pullulans & BCRC 31981, BCRC 32064, BCRC 32065 & 3 \\
\hline Chaetomium cochlioides & BCRC 31605, BCRC 30523, BCRC 31771 & 7 \\
\hline Chaetomium funicola & CBS 973.73, CBS 378.77 & 2 \\
\hline Chaetomium globosum & CBS 142.88, CBS 766.96 & 2 \\
\hline Cladosporium cladosporioides & BCR 32925, BCRC 30812, BCRC 32887 & 3 \\
\hline Mucor racemosus & BCRC 30186, BCRC 32586, BCRC 30896 & 3 \\
\hline Paecilomyces variotii & BCRC 30562, CBS 112279, CBS 370.70 & 3 \\
\hline Penicillium brevicompactum & BCRC 31258, BCRC 31259, BCRC 33336 & 3 \\
\hline Penicillium chrysogenum & BCRC 30564', BCRC 30563, BCRC 30568, BCRC 30569 & 4 \\
\hline Penicillium corylophilum & BCRC 31555, BCRC 32015, BCRC 32620 & 3 \\
\hline Rhizopus stolonifer & BCRC 31135, BCRC 31142, BCRC 31633 & 3 \\
\hline Scopulariopsis brevicaulis & ATCC 7903, ATCC 62614, BCRC 31751 & 3 \\
\hline Scopulariopsis chartarum & CBS 522.69, CBS 897.68, CBS 410.76 & 3 \\
\hline Stachybotrys chartarum & BCRC 32551, BCRC 32552, BCRC 32554, BCRC 32818, BCRC32819, BCRC 32820 & 6 \\
\hline Trichoderma viride & BCRC 32054, BCRC 3312, BCRC 33458 & 3 \\
\hline Total strain & & 77 \\
\hline
\end{tabular}

${ }^{a}$ ATCC, American Type Culture Collection, Manassas, Virginia, USA; BCRC, Bioresources Collection and Research Center, Hsinchu, Taiwan, Republic of China; CBS, Centraalbureau voor Schimmelcultures, Utrecht, The Netherlands.

Type strain. 
$50 \mu \mathrm{l}$ consisting of $10 \mathrm{mM}$ Tris- $\mathrm{HCl}(\mathrm{pH} 8.3), 0.08 \%$ Nonidet P-40 (Sigma-Aldrich, St. Louis, Minnesota, USA), $50 \mathrm{mM} \mathrm{KCl}, 1.5 \mathrm{mM} \mathrm{MgCl}_{2}, 0.8 \mathrm{mM}$ deoxynucleoside triphosphates (0.2 mM each), 1.2 U of Taq DNA polymerase, $0.7 \mu \mathrm{M}$ (each) of the forward and reverse primers, and 1 to 5 ng of DNA template. PCR was carried out using the following conditions: initial denaturation at $94^{\circ} \mathrm{C}$ for $3 \mathrm{~min}$; 35 cycles of denaturation $\left(94^{\circ} \mathrm{C}, 1 \mathrm{~min}\right)$, annealing $\left(60^{\circ} \mathrm{C}\right.$, $1 \mathrm{~min})$, and extension $\left(72^{\circ} \mathrm{C}, 1 \mathrm{~min}\right)$; and a final extension step at $72^{\circ} \mathrm{C}$ for $7 \mathrm{~min}$. PCR products were purified and sequenced by using an ABI Prism 377 automated DNA sequencer (Applied Biosystems, Taipei, Taiwan) with a BigDye Terminator cycle sequencing kit (version 3.1; Applied Biosystems).

\section{Design of oligonucleotide probes}

Species- or group-specific oligonucleotide probes (18- to 30 -mers) were designed from the ITS 1 or ITS 2 regions based on sequences in the GenBank database (Table 2) or on sequences determined in this study. The positive control probe was designed from a conserved region in the 5.8S rRNA gene [26]. The designed probes were checked for melting temperature, secondary structure, and GC content by using the Vector NTI Advance 9 (Invitrogen, Carlsbad, California, USA), and checked for potential cross-reactivity with other species in GenBank by using the BLASTN program. A total of 25 probes, including 11 previously described ones [19,26,30], were used to fabricate the oligonucleotide array on nylon membrane. Ten bases of thymine were added to the 3' ends of probes that exhibited weak hybridization signals after preliminary testing [27]. An irrelevant oligonucleotide (16 bases) labelled with a digoxigenin molecule at the 5 ' end was used as a position marker on the array.

\section{Fabrication of arrays}

The array $(0.8 \times 0.7 \mathrm{~cm})$ contained 72 dots ( 9 by 8 dots), including 50 dots for species identification (duplicate dots of each of the 25 fungal-specific probes), 5 dots for negative control (probe code $\mathrm{NC}$, tracking dye only), 2 dots for positive control (probe code PC), and 15 dots for the position marker (probe code M) (Figure 1). The oligonucleotide probes $(10 \mu \mathrm{M})$ were drawn into wells of 96-well microtiter plates, and spotted onto positively charged nylon membrane (Roche, Mannheim, Germany) as described previously [28]. The layout of all probes on the array is shown in Figure 1.

\section{Array hybridization}

The ITS region of a fungus was amplified by PCR using the forward primers (ITS1 and ITS5) and reverse primer (ITS4) as described in the previous section, with each primer being labelled with a digoxigenin molecule at the 5 ' end. The reagents and procedures for prehybridization, hybridization $\left(55^{\circ} \mathrm{C}\right.$ for $\left.90 \mathrm{~min}\right)$, and color development using enzyme-conjugated anti-digoxigenin antibodies were previously described [28]. The hybridized spots (400 $\mu \mathrm{m}$ in diameter) could be read by the naked eye. A strain was identified as one of the species listed in Table 1 when both the positive control probe and the species-specific probe (or at least one of the multiple probes designed for a species) were hybridized (Table 2). The images of the hybridization pattern were captured by a scanner (PowerLook 3000; UMAX, Taipei, Taiwan).

\section{Isolation and identification of airborne fungi}

Air samples were collected from three places (one hospital, one research laboratory, and one government office). The QuickTake 30 BioStage Pump kit (SKC Inc., Eighty Four, Pennsylvania, USA) was used to collect air samples. Fungal spores were collected on malt extract agar and Saubouraud dextrose agar for $3 \mathrm{~min}$ at a flow rate of 28.3 $\mathrm{L} / \mathrm{min}$. Spores trapped on agar plates were grown at $25^{\circ} \mathrm{C}$ for 3-7 days. Seventy colonies grown on agar plates were selected for identification by the array. The species names of the fungi identified by the array were further verified by sequencing of the ITS region and the D1/D2 domain of the large-subunit RNA gene; sequences in the two regions were found to be highly specific for a fungal species $[29,30]$. Species were identified by searching databases using the BLAST sequence analysis tool in the National Center for Biotechnology Information. If the result of array hybridization was in accordance with that of either ITS or D1/D2 domain sequencing, the identification made by the array was considered to be correct.

\section{Determination of detection limit}

Detection limit was the lowest amount of fungal DNA that could be detected by the array. Serial 10 -fold dilutions of DNAs of Aspergillus fumigatus BCRC 30502 and $A$. versicolor BCRC 31488 were used to determine the detection limits.

\section{Results}

\section{Probe design}

Initially, about 100 probes (data not shown) were designed to identify the 21 species listed in Table 1 . Through extensive screening, many probes cross-reacted with heterologous species or produced weak hybridization signals with homologous species. Finally, 25 probes were selected for fabrication of the array (Table 2); these probes included 11 oligonculeotides published in our previous studies $[19,26,30]$. One or multiple probes were designed to identify a single species, depending on the availability of divergent sequences in the ITS region (Table 2). For most species, a single probe was enough to identify an individual 
Table 2 Oligonucleotide probes used to identify airborne fungi

\begin{tabular}{|c|c|c|c|c|c|c|}
\hline \multirow[t]{2}{*}{ Microorganism } & \multicolumn{4}{|c|}{ Probe } & \multirow[b]{2}{*}{ Location $^{c}$} & \multirow[b]{2}{*}{$\begin{array}{l}\text { GenBank } \\
\text { accession no }\end{array}$} \\
\hline & Code $^{a}$ & Sequence $\left(5^{\prime}-3^{\prime}\right)^{b}$ & $\begin{array}{l}\text { Length } \\
\text { (nt) }\end{array}$ & $T_{m}\left({ }^{\circ} \mathrm{C}\right)$ & & \\
\hline Acremonium strictum $^{\mathrm{d}}$ & Acstr2-5 & CTGCGTAGTAGCACAACCTCGCAtttttttttt & 23 & 59.1 & $431-453(2)$ & AJ621771 \\
\hline Alternaria alternata ${ }^{\mathrm{e}}$ & Alalt3 & CGCACTCTCTATCAGCAAAGGTCTAGCATC & 30 & 63.5 & $461-490(2)$ & AY625056 \\
\hline Aspergillus flavus $\mathrm{e}^{\mathrm{e}}$ & Asfla4 & CGAACGCAAATCAATCTIITICCAGGT & 27 & 63.1 & $512-538(2)$ & AY373848 \\
\hline Aspergillus fumigatus $^{\mathrm{e}}$ & $\underset{1}{\text { Asfum2- }}$ & GCCAGCCGACACCCAACTTTATTITTCTAAtttttttttt & 30 & 65.4 & $213-242(2)$ & AY230140 \\
\hline Aspergillus niger ${ }^{\mathrm{e}}$ & Asnig2 & ACGTTTCCAACCATTCTITCCAGGT & 26 & 60.9 & $517-542(2)$ & AY373852 \\
\hline Aspergillus versicolor ${ }^{\mathrm{e}}$ & Asver4 & ACGTCTCCAACCATTITCTTCAGGT & 25 & 58.2 & $486-510(2)$ & AY830119 \\
\hline Aureobasidium pullulans ${ }^{\mathrm{e}}$ & Aupul2 & ATTTCTAACAACGCTCTITGGGTCGGTACG & 30 & 65.5 & $454-483(2)$ & AF121283 \\
\hline Aureobasidium pullulans $^{\mathrm{e}}$ & Aupul3 & TCAAAGGAGAGGACTTCTGCCGACTGAAAC & 30 & 66.2 & $456-485(2)$ & AY139395 \\
\hline Aureobasidium pullulans ${ }^{\mathrm{e}}$ & Aupul4 & GGCGTAGTAGAATTTATTCGAACGTCTGTC & 30 & 60.7 & $428-457(2)$ & AY139395 \\
\hline $\begin{array}{c}\text { Chaetomium cochliodes/C. } \\
\text { globosum/C. funicola }\end{array}$ & Chcgf1 & GGCCTCTCTGAGTCTTCTGTACTGAATAAG & 30 & 58.8 & $157-186(1)$ & AJ279450 \\
\hline Cladosporium cladosporioides & Ccla2-2 & CGGGAGGCTACGCCGTAAAtttttttttt & 19 & 57.4 & $470-488(2)$ & AY361994 \\
\hline Mucor racemosus & Mrac2-1 & GGGCCTCTCGATCTGTATAGATCTTttttttttt & 25 & 55.3 & $573-597(2)$ & AY625074 \\
\hline Mucor racemosus & Mrac3-1 & TAGATCTTGAAATCCCTGAAATTTACTtttttttttt & 27 & 52.7 & $590-616(2)$ & AY625074 \\
\hline Paecilomyces variotiif & Pavar2 & CCGAAGACCCCTSGAACGCtttttttttt & 19 & 59.0 & $155-173(1)$ & AY373941 \\
\hline Penicillium brevicompactum & Pbre1-1 & ACCCGCTTTGTAGGACTGCCCGtttttttttt & 22 & 63.0 & $439-461(2)$ & AY484922 \\
\hline Penicillium chrysogenum & Pchr1-1 & TCAACCCAAATTITTATCCAGGtttttttttt & 22 & 52.6 & $482-503(2)$ & DQ674380 \\
\hline Penicillium corylophilum & $\begin{array}{l}\text { Pcor1- } \\
\text { 2R }\end{array}$ & CGCGGGCCAGAGGGCAGAtttttttttttt & 18 & 63.9 & $102-119(1)$ & AF034456 \\
\hline Penicillium corylophilum & $\begin{array}{l}\text { Pcor2- } \\
2 R\end{array}$ & CGCGGGCCAGAGGGCAGAAGttttttttttt & 20 & 65.6 & $100-119(1)$ & AF033450 \\
\hline Rhizopus stolonifer & Rsto4 & AAAGGCGGTTAATGGTATCCAACAAATtttttttttt & 27 & 60.0 & $246-272(1)$ & AB113023 \\
\hline \multirow[t]{2}{*}{ Scopulariopsis chartarum } & Scchal & TCTTCATACCCATTTGTGAACACTACCCtttttttttt & 28 & 59.4 & $41-68(1)$ & AY625066 \\
\hline & $\begin{array}{c}\text { Sccha4- } \\
1\end{array}$ & AGTAAAGCACCTCGCATCGGGTCCtttttttttt & 24 & 63.2 & $497-520(2)$ & AY625066 \\
\hline Scopulariopsis brevicaulis ${ }^{\mathrm{e}}$ & $\begin{array}{c}\text { Scbre3- } \\
1\end{array}$ & TGCGTAGTAGATCCTACATCTCGCATCGtttttttttt & 28 & 62.4 & $500-527(2)$ & AY625065 \\
\hline Stachybotrys chartarum & Scha1-3 & CAGTATTCTCTGAGTGGGAAACGCAAAtttttttttt & 27 & 60.7 & $485-511(2)$ & AF081468 \\
\hline Stachybotrys chartarum & Scha1-4 & AGTATTCTCTGAGTGGTAAACGCAAAtttttttttt & 26 & 54.8 & $157-181(1)$ & AY095976 \\
\hline Trichoderma viride & Tvir2-1 & AACCAAACTCTITCTGTAGTCCCCTCtttttttttt & 26 & 56.5 & $111-136(1)$ & AY380909 \\
\hline Positive control ${ }^{9}$ & PC & GCATCGATGAAGAACGCAGCttttttttt & 20 & 55.7 & $200-219$ & EF134625 \\
\hline
\end{tabular}

aligonucleotide probes are arranged on the array as indicated in Figure 1.

${ }^{b}$ Multiple bases of thymine, indicated by " $\mathrm{t}$ ", were added to the $3^{\prime}$ end of the probe. The underlined nucleotide indicates a single mismatch base that was intentionally incorporated into the probe to avoid cross-hybridization.

'The location of probe is shown by the nucleotide number of either ITS 1 or ITS 2 ; the number (1 or 2) in parenthesis indicates the ITS region from which the probe was designed.

dProbe modified from a previous study (19).

eProbes designed in a previous study (19).

fProbe designed in a previous study (26).

${ }^{9}$ The positive control probe was designed from a conserved region of the 5.8S rRNA gene (30).

microorganism. But one probe (code Chcgf1) was used to identify a group of three closely related species (Chaeotomium cochlioides, C. globosum, and C. fumicola) due to high interspecies similarities of the ITS sequences among these species. Conversely, some fungi displayed high intraspecies sequence divergence in the ITS regions and hence multiple probes were constructed to identify a single species. For example, two probes were used to identify each of the following species: Mucor racemosus, Penicillium corylophilum, Scopulariopsis chartarum, and
Stachybotrys chartarum, and three probes were synthesized to identify Aureobasidium pullulans (Table 2).

\section{Sensitivity and specificity of the array}

A total of 139 reference strains, including 73 target and 66 nontarget strains, were analyzed by the array. The hybridization patterns of different fungal species are shown in Figure 2. Of the 73 target strains, 72 (98.6\%) were correctly identified to the species or group level by the array, with one strain (Trichoderma viride BCRC 


\begin{tabular}{|c|c|c|c|c|c|c|c|c|c|}
\hline & $\mathbf{1}$ & $\mathbf{2}$ & $\mathbf{3}$ & $\mathbf{4}$ & $\mathbf{5}$ & $\mathbf{6}$ & $\mathbf{7}$ & $\mathbf{8}$ & $\mathbf{9}$ \\
\hline A & Acstr2-5 & Acstr2-5 & Alalt3 & Alalt3 & M & Asfla4 & Asfla4 & Asfum2-1 & Asfum2-1 \\
\hline B & Asnig2 & Asnig2 & Aupul2 & Aupul2 & M & Aupul3 & Aupul3 & Aupul4 & Aupul4 \\
\hline C & Asver4 & Asver4 & Ccla2-2 & Ccla2-2 & M & Chcgf1 & Chcgf1 & NC & NC \\
\hline D & Mrac2-1 & Mrac2-1 & Mrac3-1 & Mrac3-1 & M & Pavar2 & Pavar2 & PC & PC \\
\hline E & M & M & M & M & NC & M & M & M & M \\
\hline F & Pbre1-1 & Pbre1-1 & NC & NC & M & Pcor1-2R & Pcor1-2R & Pcor2-2R & Pcor2-2R \\
\hline G & Pchr1-1 & Pchr1-1 & Rsto4 & Rsto4 & M & Scha1-3 & Scha1-3 & Scha1-4 & Scha1-4 \\
\hline H & Sccha1 & Sccha1 & Sccha4-1 & Sccha4-1 & M & Scbre3-1 & Scbre3-1 & Tvir2-1 & Tvir2-1 \\
\hline
\end{tabular}

Figure 1 Layout of oligonucleotide probes on the array $(0.8 \times 0.7 \mathrm{~cm}, 9$ by 8 dots). The probe "PC" was a positive control and the probe "NC" was a negative control (tracking dye only). The probe " $\mathrm{M}$ ", a position marker, was an irrelevant probe labeled with a digoxigenin molecule at the $5^{\prime}$ end. The corresponding species names and sequences of all probes are listed in Table 2. All probes used for fungal identification were spotted on the array in duplicate.

32054) being not identified (only the positive control was hybridized). Discrepancy analysis revealed that the strain BCRC 32054 had an ITS 1 and ITS 2 sequence similarity of $100 \%$ with Trichoderma harzianum, while the corresponding sequence similarities were only $78.7 \%$ and $87.2 \%$, respectively, with a reference sequence of Trichoderma viride in GenBank (accessing no. X93978). It was obvious that Trichoderma viride BCRC 32054 was a misidentification of Trichoderma harzianum, a nontarget species in this study. Therefore, the sensitivity of the array was $100 \%(72 / 72)$. In addition, a collection of 66 nontarget strains (66 species) were used for specificity testing of the array (additional file 1). No crosshybridization was observed for any strain analyzed and a specificity of $100 \%$ (66/66) was obtained.

\section{Detection limit of the array}

Serial 10-fold dilutions of DNAs extracted from two strains (Aspergillus fumigatus BCRC 30502 and A. versicolor BCRC 31488) were used to determine the detection limits. For both strains, the detection limit of the array was $10 \mathrm{pg}$ genomic DNA per assay; this amount of DNA was approximately equal to 270 cells (37 fg of DNA per cell of Candida albicans) [13].

\section{Identification of fungal strains isolated from air samples}

The array was used to identify 70 fungal isolates recovered from the air samples in three buildings including one hospital (24 strains), one research laboratory (11 strains), and one office (35 strains). Among the 70 strains, 27 were identified to species level by the array, and 43 strains were not identified (nontarget species). The identified airborne fungi were Aspergillus fumigatus and A. versicolor (from a hospital), A. niger and A. versicolor (from a laboratory), and Alternaria alternata, Aspergillus flavus, Cladosporium cladosporioides, and Penicillium chrysogenum (from an office) (Table 3). Among the 27 strains identified by hybridization, 26 were correctly identified, as revealed by their morphological characteristics and sequencing of the ITS and ribosomal D1/D2 domains of the rRNA operons (Table 3). A strain (no. 12) was misidentified as Acremonium strictum by the array, since the ITS sequences demonstrated that the strain was Acremonium implicatum, a nontarget species. The remaining 43 non-identified strains from air samples belonged to nontarget species, as evidenced by their ITS and D1/D2 sequences (additional file 2). Some nontarget strains were only identified to the genus level by DNA sequencing since there were no corresponding ITS or D1/D2 sequence entries in the public database. Based on these results, the sensitivity and specificity of the array for identification of airborne fungi were $100 \%(26 / 26)$ and $97.7 \%(43 / 44)$, respectively. Among the 70 isolates recovered from air samples, 26 (37.1\%) have potentials to cause allergy or adverse health problems in susceptible individuals.

\section{Discussion}

In this study, an oligonucleotide array was developed to identify 21 species of airborne fungi that are of health concern (Table 1). High sensitivity and specificity of the array were demonstrated by testing a collection of 138 reference strains and 70 isolates from air samples. Comparing with glass chip, the current membrane array is relatively simple, time-saving, and the test cost was quite low. In addition, only minimal instrumentation 


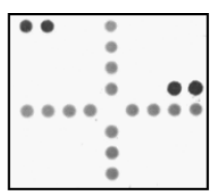

Acremonium strictum BCRC 32239

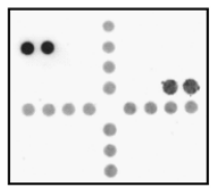

Aspergillus niger BCRC 31130

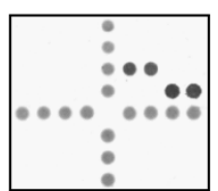

Chaetomium cochlioides BCRC 31771

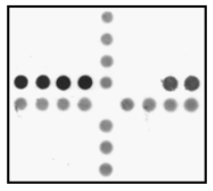

Mucor racemosus BCRC 32586

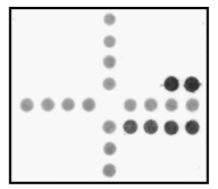

Penicillium corylophilum BCRC 32015

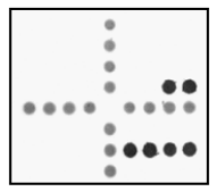

Stachybotrys chartarum BCRC 32551

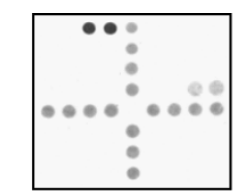

Alternariaalternata BCRC 32888

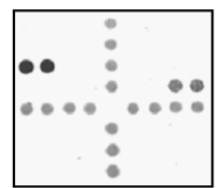

Aspergillus versicolor BCRC 31123

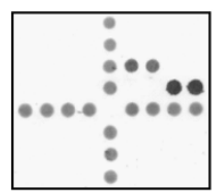

Chaetomium funicola CBS 973.73

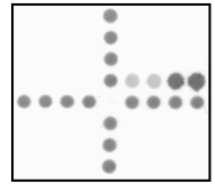

Paecilomyces variotii CBS 370.70

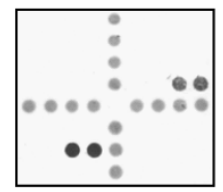

Rhizopus stolonifer BCRC 31135

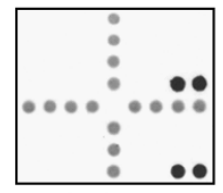

Trichoderma viride BCRC 33129

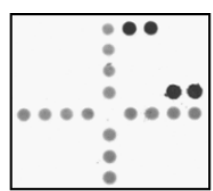

Aspergillus flavus BCRC 30165

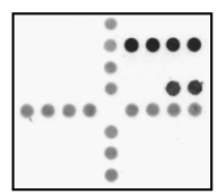

Aureobasidium pullulans BCRC 31981

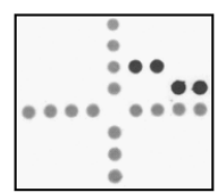

Chaetomium globosum BCRC 31605

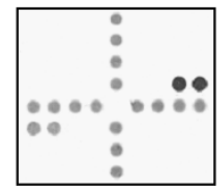

Penicillium brevicompactum Penicillium chrysogenum BCRC 31258

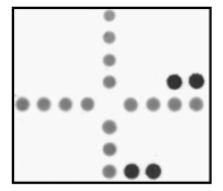

Scopulariopsis brevicaulis ATCC 7903

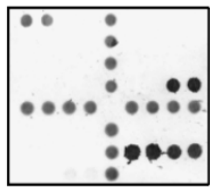

Acremonium strictum Stachybotrys chartarum

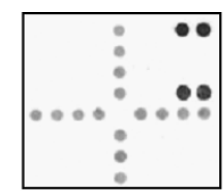

Aspergillus fumigatus BCRC 30502

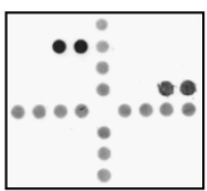

Aureobasidium pullulans BCRC 32064

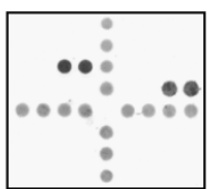

Cladosporium cladosporioides BCRC 30812

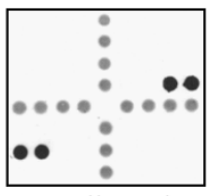

BCRC 30564

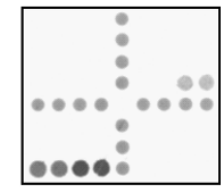

Scopulariopsis chartarum CBS 897.68

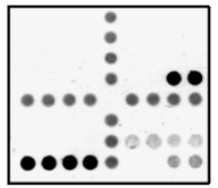

Scopulariopsis chartarum Stachybotrys chartarum Trichoderma viride

Figure 2 Hybridization patterns of 21 species of fungi on the array. The corresponding probes hybridized on the array are indicated in Figure 1, and the corresponding sequences of the hybridized probes are shown in Table 2. All probes used for fungal identification were spotted on the array in duplicate. The last two panels shows the simultaneous hybridization of two (Acremonium strictum and Stachybotrys chartarum) and three species (Scopulariopsis chartarum, Stachybotrys chartarum, and Trichoderma viride), respectively, on a single array.

(a shaker and an incubator) is required for hybridization. The whole procedure for fungal detection by the array can be finished within a working day $(8 \mathrm{~h})$, starting from isolated colonies. The prominent feature of the current method is the use of a standardized protocol encompassing DNA extraction, ITS amplification, and membrane hybridization. The examinations of fungal reproductive structures, which are essential for classical identification, are not required by the present method.

In this study, one or multiple probes were designed to identify a single species, depending on the availability of divergent sequences in the ITS region (Table 2). The 
Table 3 Identification of fungi isolated from indoor air by the array and by sequence analysis of the ITS and D1/D2 domain

\begin{tabular}{|c|c|c|c|c|}
\hline \multirow[t]{2}{*}{ Strain no. ${ }^{a}$} & \multicolumn{4}{|c|}{ Species identification by } \\
\hline & Array hybridization & ITS sequence $(\%)^{a}$ & D1/D2 sequence $(\%)^{a}$ & Best match \\
\hline 1 & Cladosporium cladosporioides & Cladosporium cladosporioides (99.4) & Cladosporium cladosporioides (99.6) & Cladosporium cladosporioides \\
\hline 3 & Aspergillus versicolor & Aspergillus versicolor (100) & Aspergillus versicolor (99.0) & Aspergillus versicolor \\
\hline 5 & Aspergillus niger & Aspergillus niger (100) & Aspergillus niger (100) & Aspergillus niger \\
\hline 12 & Acremonium strictum $^{b}$ & Acremonium implicatum (100) & Acremonium sp. (97.8) & Acremonium implicatum \\
\hline 13 & Cladosporium cladosporioides & Cladosporium cladosporioides (100) & Cladosporium cladosporioides (100) & Cladosporium cladosporioides \\
\hline 28 & Alternaria alternata & Alternaria alternata (100) & Alternaria alternata (98.6) & Alternaria alternata \\
\hline 33 & Aureobasidium pullulans & Aureobasidium pullulans (100) & Aureobasidium pullulans (98.2) & Aureobasidium pullulans \\
\hline 35 & Aspergillus flavus & Aspergillus flavus (100) & Aspergillus flavus (100) & Aspergillus flavus \\
\hline 38 & Aspergillus flavus & Aspergillus flavus (99.8) & Aspergillus flavus (99.8) & Aspergillus flavus \\
\hline 41 & Cladosporium cladosporioides & Cladosporium cladosporioides (100) & Cladosporium cladosporioides (99.6) & Cladosporium cladosporioides \\
\hline 42 & Cladosporium cladosporioides & Cladosporium cladosporioides (99.8) & Cladosporium cladosporioides (99.8) & Cladosporium cladosporioides \\
\hline 43 & Cladosporium cladosporioides & Cladosporium cladosporioides (100) & Cladosporium cladosporioides (99.8) & Cladosporium cladosporioides \\
\hline 44 & Cladosporium cladosporioides & Cladosporium cladosporioides (100) & Cladosporium cladosporioides (100) & Cladosporium cladosporioides \\
\hline 45 & Cladosporium cladosporioides & Cladosporium cladosporioides (100) & Cladosporium cladosporioides (99.6) & Cladosporium cladosporioides \\
\hline 47 & Cladosporium cladosporioides & Cladosporium cladosporioides (100) & Cladosporium cladosporioides (99.6) & Cladosporium cladosporioides \\
\hline 48 & Cladosporium cladosporioides & Cladosporium cladosporioides (100) & Cladosporium cladosporioides (99.6) & Cladosporium cladosporioides \\
\hline 49 & Aspergillus versicolor & Aspergillus versicolor (99.8) & Aspergillus versicolor (99.3) & Aspergillus versicolor \\
\hline 50 & Aspergillus versicolor & Aspergillus versicolor (100) & Aspergillus versicolor (99.3) & Aspergillus versicolor \\
\hline 52 & Aspergillus versicolor & Aspergillus versicolor (99.8) & Aspergillus versicolor (99.1) & Aspergillus versicolor \\
\hline 55 & Aspergillus versicolor & Aspergillus versicolor (100) & Aspergillus versicolor (99.8) & Aspergillus versicolor \\
\hline 56 & Aspergillus versicolor & Aspergillus versicolor (100) & Aspergillus versicolor (98.6) & Aspergillus versicolor \\
\hline 58 & Aspergillus versicolor & Aspergillus versicolor (100) & Aspergillus versicolor (99.1) & Aspergillus versicolor \\
\hline 59 & Aspergillus versicolor & Aspergillus versicolor (100) & Aspergillus versicolor (99.3) & Aspergillus versicolor \\
\hline 60 & Aspergillus versicolor & Aspergillus versicolor (100) & Aspergillus versicolor (99.1) & Aspergillus versicolor \\
\hline 64 & Aspergillus niger & Aspergillus niger (99.8) & Aspergillus niger (99.1) & Aspergillus niger \\
\hline 67 & Aspergillus niger & Aspergillus niger (99.8) & Aspergillus niger (99.6) & Aspergillus niger \\
\hline 68 & Aspergillus niger & Aspergillus niger (99.8) & Aspergillus niger (100) & Aspergillus niger \\
\hline
\end{tabular}

aalues in parentheses are percentages of sequence similarities of the test isolates with the best-scoring sequences in the GenBank database.

${ }^{\mathrm{b}}$ The strain was misidentified by array hybridization.

advantage of using multiple probes is the increased coverage of different strains of a species, but the disadvantage is the potential decrease of specificity due to the unpredictable cross-hybridizations caused by other irrelevant fungal species. The $T_{m}$ (melting temperature) values of probes used in this study ranged from 52.6 to $66.2^{\circ} \mathrm{C}$, with some probes had $T_{m}$ lower than the hybridization temperature $\left(55^{\circ} \mathrm{C}\right)$ (Table 2$)$. However, clear signals were obtained for all target strains tested (Figure 2). Volokhov et al. [31] also reported the successful use of probes having $T_{m}$ values lower than the hybridization temperature for bacterial identification. The addition of several thymine bases to the end of a probe (Table 2) had the benefit of reducing steric hindrance between target DNA and the probe immobilized on a solid support [27]

In a previous study, an array targeting the $18 \mathrm{~S}$ rRNA gene was developed to identify airborne fungi [24]. Since the $18 \mathrm{~S}$ rRNA genes are highly conserved in closely related species, therefore species identification was based on hybridization patterns involving a combination of multiple probes rather than on species-specific probes [24]. The advantage of the current study is that all species were discretely identified by specific probes and the reading of results is very straightforward. The successful design of different probes was based on the known ITS sequences (Table 2), and multiple sequence alignment (interspecies and intraspecies) played an important role in finding out the regions that could be utilized for probe design. The present array is a powerful tool for the identification of important airborne fungi that may cause health problems in susceptible individuals. The array has the potential to be continually extended by including more probes, without significant increase of the cost or complexity. The current method permits a shorter time to achieve results as well as the correct identification of morphologically indistinguishable species. 
Furthermore, the array was able to identify multiple fungal species at the same time as demonstrated in Figure 2 (the last two arrays). The DNAs of colonies from two (Acremonium strictum and Stachybotrys chartarum) or three species (Scopulariopsis chartarum, Stachybotrys chartarum, and Trichoderma viride) on agar plates were extracted in a tube, amplified by PCR, and hybridized to an array. All individual species were simultaneously identified. Furthermore, we also tried to directly detect fungi by trapping airborne spores in a buffer, followed by centrifugation, DNA extraction, and array hybridization. However, comparing with culture, the direct method was less sensitive and this might be due to the limited numbers of spores collected (data not shown). It is anticipated that by improving the sampling method, DNA extraction efficiency, and using nested PCR [26], the current array may have a potential to directly detect airborne fungi without an initial cultivation step. A possible method would be that PCR can be directly performed on the collected fungal spores, omitting the DNA extraction step that may lead to a significant loss of DNA for a very small sample. However, further investigation is needed to verify this hypothesis.

\section{Conclusions}

Identification of airborne fungi by the array is highly reliable and accurate. The method can be used as an effective alternative to the conventional identification methods. The current array can greatly contribute to decipher the relationship between airborne fungi and adverse health effects.

\section{Additional material}

Additional file 1: Additional Table 1: Nontarget fungi used for specificity testing of the array. 66 strains of nontarget fungi used to test the specificity of the array developed in this study

Additional file 2: Additional Table 2: Fungal strains not identified by the array and identification of these strains by sequencing of the ITS and D1/D2 domain. Identification of 44 air fungal isolates not identified by the array by sequencing of the ITS and D1/D2 domain

\section{Acknowledgements}

This study was supported by grants (EPA-98-E3S5-02-01 and DOH100-TD-B111-002) from the Environmental Analysis Laboratory, Environmental Protection Administration, Taiwan and the Multidisciplinary Center of Excellence for Clinical Trial and Research, Taiwan.

\section{Author details}

${ }^{1}$ Environmental Analysis Laboratory, Environmental Protection Administration, Zhongli, Taiwan. ${ }^{2}$ Department of Medical Laboratory Science and Biotechnology, College of Medicine, National Cheng Kung University, Tainan, Taiwan.

\section{Authors' contributions}

WH coordinated the project. SS and LS performed data analysis, specimen collection and testing. TCC contributed to study design, coordinated the experiment, and drafted the manuscript. All authors read and approved the final manuscript.

\section{Competing interests}

The authors declare that they have no competing interests.

Received: 24 December 2010 Accepted: 13 April 2011

Published: 13 April 2011

\section{References}

1. Bush RK, Portnoy JM: The role and abatement of fungal allergens in allergic diseases. J Allergy Clin Immunol 2001, 107:430-440.

2. Bush RK, Portnoy JM, Saxon A: The medical effects of mold exposure. J Allergy Clin Immunol 2006, 107:326-333.

3. Hodgson MJ, Moery $P$, Leung WY, Morrow L, Miller D, Jarvis BB, Robbins $H$, Halsey JF, Storey E: Building-associated pulmonary disease from exposure to Stachybotrys chartarum and Aspergillus versicolor. J Occup Environ Med 1998, 40:241-249.

4. Su HJ, Wu PC, Chen HL, Lee FC, Lin LL: Exposure assessment of indoor allergens, endotoxin, and airborne fungi for homes in southern Taiwan. Environ Res 2001, 85:135-144.

5. Anandan C, Nurmatov U, van Schayck OC, Sheikh A: Is the prevalence of asthma declining? Systematic review of epidemiological studies. Allergy 2010, 65:152-167.

6. García-Marcos L, Quirós AB, Hernánde GG, Guillén-Grima F, Díaz CG, Ureña IC, Pena AA, Monge RB, Suárez-Varela MM, Varela AL, Cabanillas PG, Garrido JB: Stabilization of asthma prevalence among adolescents and increase among school children (ISAA phases I and III) in Spain. Allergy 2004, 59:1301-1307.

7. Wang XS, Tan TN, Shek LP, Chng SY, Hia CP, Ong NB, Ma S, Lee BW, Goh DY: The prevalence of asthma and allergies in Singapore: data from two ISAAC surveys seven years apart. Arch Dis Child 2004, 89:423-426.

8. Ritz N, Ammann R: Risk factors for allergic bronchopulmonary aspergillosis and sensitization to Aspergillus fumigatus in patients with cystic fibrosis. Eur J Pediatr 2005, 164:577-582.

9. Simon-Nobbe B, Denk U: The spectrum of fungal allergy. Int Ach Allergy Immunol 2008, 145:58-68.

10. Larone DH: Medically Important Fungi: A Guide to Identification. 4 edition. Washington DC: ASM Press; 2002.

11. Brunner K, Zeilinger S, Mach RL: Environmental and Microbial Relationships. 2 edition. Heidelberg, Germany: Springer-Verlag; 2007.

12. Chen SCA, Halliday CL, Meyer W: A review of nucleic acid-based diagnostic tests for systemic mycoses with an emphasis on polymerase chain reaction-based assays. Med Mycol 2002, 40:333-357.

13. Chang HC, Leaw SN, Huang AH, Wu TL, Chang TC: Rapid identification of yeasts in positive blood cultures by a multiplex PCR method. J Clin Microbiol 2001, 39:3466-3471

14. Martin C, Roberts D, van Der Weide M, Rossau R, Jannes G, Smith T, Maher M: Development of a PCR-based line probe assay for identification of fungal pathogens. J Clin Microbiol 2000, 38:3735-3742.

15. Velegraki A, Kambouris ME, Skiniotis G, Savala M, Mitroussia-Ziouva A, Legakis NJ: Identification of medically significant fungal genera by polymerase chain reaction followed by restriction enzyme analysis. FEMS Immunol Med Microbiol 1999, 23:303-312.

16. Hsu MC, Chen KW, Lo HJ, Chen YC, Liao MH, Lin YH, Li SY: Species identification of medically important fungi by use of real-time LightCycler PCR. J Med Microbiol 2003, 52:1071-1076.

17. Dannaoui E: Molecular tools for identification of Zygomycetes and the diagnosis of zygomycosis. Clin Microbiol Infect 2009, 15(Suppl 5):66-70.

18. Hall L, Wohlfiel S, Roberts GD: Experience with the MicroSeq D2 largesubunit ribosomal DNA sequencing kit for identification of filamentous fungi encountered in the clinical laboratory. J Clin Microbiol 2004, 42:622-626.

19. Hsiao CR, Huang L, Bouchara JP, Barton R, Li HC, Chang TC: Identification of medically important molds by an oligonucleotide array. J Clin Microbiol 2005, 43:3760-3768

20. Huang A, Li JW, Shen ZQ, Wang XW, Jin M: High-throughput identification of clinical pathogenic fungi by hybridization to an oligonucleotide microarray. J Clin Microbiol 2006, 44:3299-3305. 
21. Leaw SN, Chang HC, Barton R, Bouchara JP, Chang TC: Identification of medically important yeasts by an oligonucleotide array. J Clin Microbiol 2007, 45:2220-2229.

22. Leinberger DM, Schumacher U, Autenrieth IB, Bachmann TT: Development of a DNA microarray for detection and identification of fungal pathogens involved in invasive mycoses. I Clin Microbiol 2005, 43:4943-4953.

23. Li HC, Bouchara JP, Hsu MM, Barton R, Chang TC: Identification of dermatophytes by an oligonucleotide array. J Clin Microbiol 2007, 45:3160-3166

24. Wu Z, Tsumura Y, Blomquist G, Wang XR: $18 \mathrm{~S}$ rRNA gene variation among common airborne fungi, and development of specific oligonucleotide probes for the detection of fungal isolates. Appl Environ Microbiol 2003, 69:5389-5397.

25. White TJ, Bruns T, Lee S, Taylor J: PCR Protocols: A Guide to Methods and Applications San Diego: Academic Press; 1990.

26. Bouchara JP, Shieh HY, Croquefer S, Barton R, Marchais V, Pihet M, Chang TC: Development of an oligonucleotide array for direct detection of fungi in sputum samples from patients with cystic fibrosis. J Clin Microbiol 2009, 47:142-152.

27. Brown TJ, Anthony RM: The addition of low numbers of $3^{\prime}$ thymine bases can be used to improve the hybridization signal of oligonucleotides for use within arrays on nylon supports. J Microbiol Method 2000, 42:203-207.

28. Tung SK, Teng LJ, Vaneechoutte M, Chen HM, Chang TC: Array-based identification of species of the genera Abiotrophia, Enterococcus, Granulicatella and Streptococcus. J Clin Microbiol 2006, 44:4414-4424.

29. Kurtzman CP, Robnett CJ: Identification of clinically important ascomycetous yeasts based on nucleotide divergence in the $5^{\prime}$ end of the large-subunit (26S) ribosomal DNA gene. J Clin Microbiol 1997. 35:216-1223

30. Leaw SN, Chang HC, Sun HF, Barton R, Bouchara JP, Chang TC: Identification of medically important yeast species by sequence analysis of the internal transcribed spacer regions. J Clin Microbiol 2006, 44:693-699.

31. Volokhov D, Rasooly A, Chumakov K, Chizhikov V: Identification of Listeria species by microarray-based assay. J Clin Microbiol 2002, 40:4720-4728.

\section{Pre-publication history}

The pre-publication history for this paper can be accessed here: http://www.biomedcentral.com/1471-2334/11/91/prepub

doi:10.1186/1471-2334-11-91

Cite this article as: Hung et al:: Rapid identification of allergenic and pathogenic molds in environmental air by an oligonucleotide array. BMC Infectious Diseases 2011 11:91.

\section{Submit your next manuscript to BioMed Central and take full advantage of:}

- Convenient online submission

- Thorough peer review

- No space constraints or color figure charges

- Immediate publication on acceptance

- Inclusion in PubMed, CAS, Scopus and Google Scholar

- Research which is freely available for redistribution

Submit your manuscript at www.biomedcentral.com/submit
Biomed Central 